\title{
ТЕПЛООБМЕН ПРИ ТЕЧЕНИИ ВОДЫ СВЕРХКРИТИЧЕСКОГО ДАВЛЕНИЯ В ТРУБЕ В РЕЖИМАХ С ИЗМЕНЕНИЕМ ТЕПЛОВОЙ НАГРУЗКИ
}

\author{
Авраменко А.А., член-корреспондент НАН Украины, Ковецкая М.М., к.т.Н., \\ Кондратьева Е.А., к.т.н., Тыринов А.И., к.т.н. \\ Институт технической теплофизики НАН Украины, ул. Желябова, 2 а, Киев, 03680, Украина
}

Представлено р результати чисельного моделювання теплообмінних процесів плину води надкритичного тиску в вертикальній трубі, що обігрівається, в нестаціонарних режимах зі зміною теплового навантаження.
Представлены результаты численного моделирования теплообменных процессов при течении воды сверхкритического давления в вертикальной обогреваемой трубе в нестационарных режимах с изменением тепловой нагрузки.
The results of numeral simulations of heat transfer processes are presented for the flow of supercritical water in the vertical heated pipe in nonstationary conditions with a modification of thermal loading.

Библ. 12 , рис. 8.

Ключевые слова: численное моделирование, вертикальная труба, сверхкритическое давление, нестационарный режим.

$G_{\mathrm{k}}$ - генерация турбулентной энергии;

$g$ - ускорение свободного падения;

$h$ - энтальпия;

$k$ - кинетическая энергия турбулентности;

$\operatorname{Pr}$ - число Прандтля;

$p$ - давление;

$q$ - плотность теплового потока;

$S$ - тензор скоростей деформации;

$s-$ постоянная, равная 0.97 ;

$T$ - температура;

$V$ - вектор скорости теплоносителя;

$w$ - скорость;

$y$ - поперечная координата;

$z$ - продольная координата;

\section{Введение}

Систематические исследования течения воды в каналах при высоких (близких к критическим) давлениях и температурах начались в середине прошлого века. Интерес к этим исследованиям не ослабевает как с точки зрения получения фундаментальных знаний о природе и свойствах флюида, так и в связи с перспективой его практического использования. В частности обсуждается вопрос использования воды сверхкритического давления в атомной энергетике для охлаждения активной зоны ядерных реакторов $\delta_{\text {nm }}-$ тензор Кронекера второго ранга;

$\varepsilon-$ скорость диссипации;

$\overrightarrow{\mathrm{k}}$ - орт;

$\mu-$ вязкость;

$\rho-$ плотность.

\section{Индексы:}

вх - входной параметр;

ж-жидкость;

eff - эффективный;

k - кинетический;

t - турбулентный;

$\varepsilon-$ относится к скорости диссипации.

IV поколения [1-3].

Однако переход на сверхкритическое давление теплоносителя в энергетике связан с рядом теплофизических проблем, одной из которых является определение безопасной области тепловых нагрузок, исключающих возникновение режимов ухудшенной теплоотдачи. Такие режимы возникают в обогреваемых каналах при течении воды сверхкритического давления в области перехода от докритической к сверхкритической температуре. Исследованию условий возникновения режимов ухудшенной теплоотдачи, 
которые сопровождаются резким локальным повышением температуры стенки канала, способным вызвать ее разрушение, уделяется большое внимание [4-7].

Исследования гидродинамики и теплообмена при течении воды сверхкритического давления в вертикальных обогреваемых трубах показали, что возникновение режимов ухудшенного теплообмена связано с изменением структуры потока, существенной деформацией профиля скорости, вызванной термическим ускорением потока вблизи стенки [8-10]. В работе [10] представлены результаты численного исследования теплообмена при течении воды сверхкритического давления в вертикальной обогреваемой трубе на основе $\mathrm{RNG} k-\varepsilon$ модели турбулентности. Расчеты, выполненные для трубы диаметром 16 мм, длиной 4 м при давлении 24,5 МПа, массовой скорости 250 кг/( $\left.\mathrm{M}^{2} \mathrm{c}\right)$, температурах на входе 200, 230, $250{ }^{\circ} \mathrm{C}$ показали, что минимальное значение параметра $q / \rho w$, при котором возникают локальные области ухудшенного теплообмена, составило 1,2 кДж/кг. Возникновение режимов ухудшенного теплообмена наблюдается в области перехода к сверхкритической температуре и связано с существенной деформацией профиля скорости.

Наиболее интересными, с точки зрения безопасности и надежности работы оборудования, являются исследования нестационарных режимов, связанных с изменением тепловой нагрузки. Экспериментальных данных об условиях возникновения и развития режимов ухудшенного теплообмена при течении воды сверхкритического давления в каналах в нестационарных режимах крайне мало. Так в экспериментах [11] отмечается самопроизвольный рост температуры стенки трубы в течении 60 с при скачкообразном увеличении тепловой нагрузки от 0,29 до $0,34 \mathrm{MBT} / \mathrm{M}^{2}$ при массовой скорости 380 кг $/ \mathrm{M}^{2} \mathrm{c}$, температуре воды на входе $360{ }^{\circ} \mathrm{C}$, давлении 24,5 МПа. Время обратного перехода от режима ухудшенного теплообмена к нормальному режиму при снижении тепловой нагрузки до значения 0,28 MBт/м² составило 28 с. Наблюдались также аномальные режимы, при которых обратный переход от режима ухудшенного теплообмена к нормальному режиму при снижении тепловой нагрузки длился гораздо дольше, чем переход к режиму ухудшенного теплообмена при увеличении тепловой нагрузки.

В работе представлены результаты численного моделирования теплообмена при течении воды сверхкритического давления в вертикальной обогреваемой трубе в переходных режимах, обусловленных изменением тепловой нагрузки.

\section{Математическая модель}

Математическая модель, описывающая нестационарный процесс, включает систему уравнений сохранения массы, количества движения и энергии теплоносителя, а также уравнения для кинетической энергии турбулентности и скорости диссипации энергии на основе RNG $k-\varepsilon$ модели турбулентности [10]

$$
\begin{aligned}
& \frac{\partial \rho}{\partial t}+\operatorname{div}(\rho V)=0 \text {, } \\
& \rho[\frac{\partial V}{\partial t}+\underbrace{s \frac{\partial \nabla\left(\mu_{t} / \rho\right)}{\partial t}}_{\mathrm{I}}+(V \cdot \nabla) V]= \\
& =-\nabla p+\nabla \cdot\left[\mu_{\mathrm{eff}}\left(\nabla V+(\nabla V)^{T}-\frac{2}{3} \nabla V \delta_{\mathrm{nm}}\right)\right]+F \overrightarrow{\mathrm{k}}, \\
& \frac{\partial \rho h}{\partial t}+\underbrace{s \frac{\partial}{\partial t} \nabla \cdot\left(\frac{\left(\mu_{t}\right)}{\sqrt{2} S} \nabla h\right)}_{\text {I }}+\nabla(\rho h V)=\nabla \cdot\left(\frac{\mu_{\text {eff }}}{\operatorname{Pr}_{\text {eff }}} \nabla h\right) \text {, }
\end{aligned}
$$

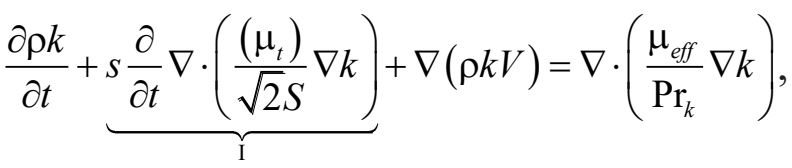

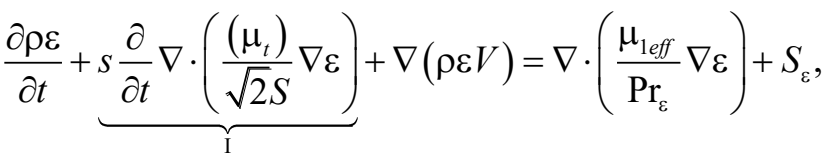

$$
\begin{aligned}
& G_{k}=\mu\left(\nabla V+(\nabla V)^{T}-\frac{2}{3} \nabla V \delta_{\mathrm{nm}}\right)^{2}, \\
& S=\frac{1}{2}\left(\frac{\partial u_{i}}{\partial x_{j}}+\frac{\partial u_{j}}{\partial x_{i}}\right), S_{\varepsilon}=\rho \frac{\varepsilon}{k}\left(C_{1} G_{k}-C_{2} \varepsilon\right), \\
& C_{1}=1,42, C_{2}=1,68,
\end{aligned}
$$

где $F=\rho g-$ подъемная сила, $\nabla-$ тензорная дивергенция, $\nabla V$ - тензорный градиент, который преобразует вектор (тензор первого ранга) в тен- 
зор второго ранга, $(\nabla V)^{T}$ - сопряженный тензорный градиент, $I$ - дополнительное слагаемое, учитывающее нестационарность турбулентных процессов, $\delta_{\mathrm{nm}}-$ тензор Кронекера второго ранга соответствующей размерности, $\mu_{\text {eff }}=\mu_{t}+\mu-$ эффективная вязкость. Значение $\operatorname{Pr}_{\text {eff }}$ определяется из уравнения

$$
\left|\frac{\operatorname{Pr}_{e f f}^{-1}-a}{\operatorname{Pr}^{-1}-a}\right|^{\frac{a+1}{a+b}}\left|\frac{\operatorname{Pr}_{e f f}^{-1}+b}{\operatorname{Pr}^{-1}+b}\right|^{\frac{b-1}{a+b}}=\frac{\mu}{\mu_{e f f}}, \text { где }
$$

$a=\frac{1}{2}\left(\sqrt{4 \frac{d-1}{d} \widetilde{A}_{d}^{-1}+1}-1\right), b=\frac{1}{2}\left(\sqrt{4 \frac{d-1}{d} \widetilde{A}_{d}^{-1}+1}+1\right)$,

$\widetilde{A}_{d}=\frac{d-1}{2(d+2)}, d-$ размерность пространства.

Теплофизические свойства воды описывались согласно формуляции IF-97 [12].

\section{Результаты расчета}

Расчеты выполнены в вертикальной обогреваемой трубе диаметром 16 мм, длиной 4 м. Ось $z$ совпадает с осью трубы, начало координат совпадает с входным сечением. В качестве начального условия использовалось решение стационарной задачи при давлении 24,5 МПа, массовой скорости 250 кг/( $\left.\mathrm{M}^{2} \mathrm{c}\right)$, температуре воды на входе $200{ }^{\circ} \mathrm{C}$.

Увеличение тепловой нагрузки

В режиме с увеличением тепловой мощности начальное значение плотности теплового потока на стенке трубы составляло 300 кВт/м². За 2 с плотность теплового потока увеличивалась до 400 кВт/м² по линейному закону и далее оставалась на этом уровне. Общее время расчета составило 60 с. На рис. 1 показано изменение во времени распределение температуры стенки по длине трубы.

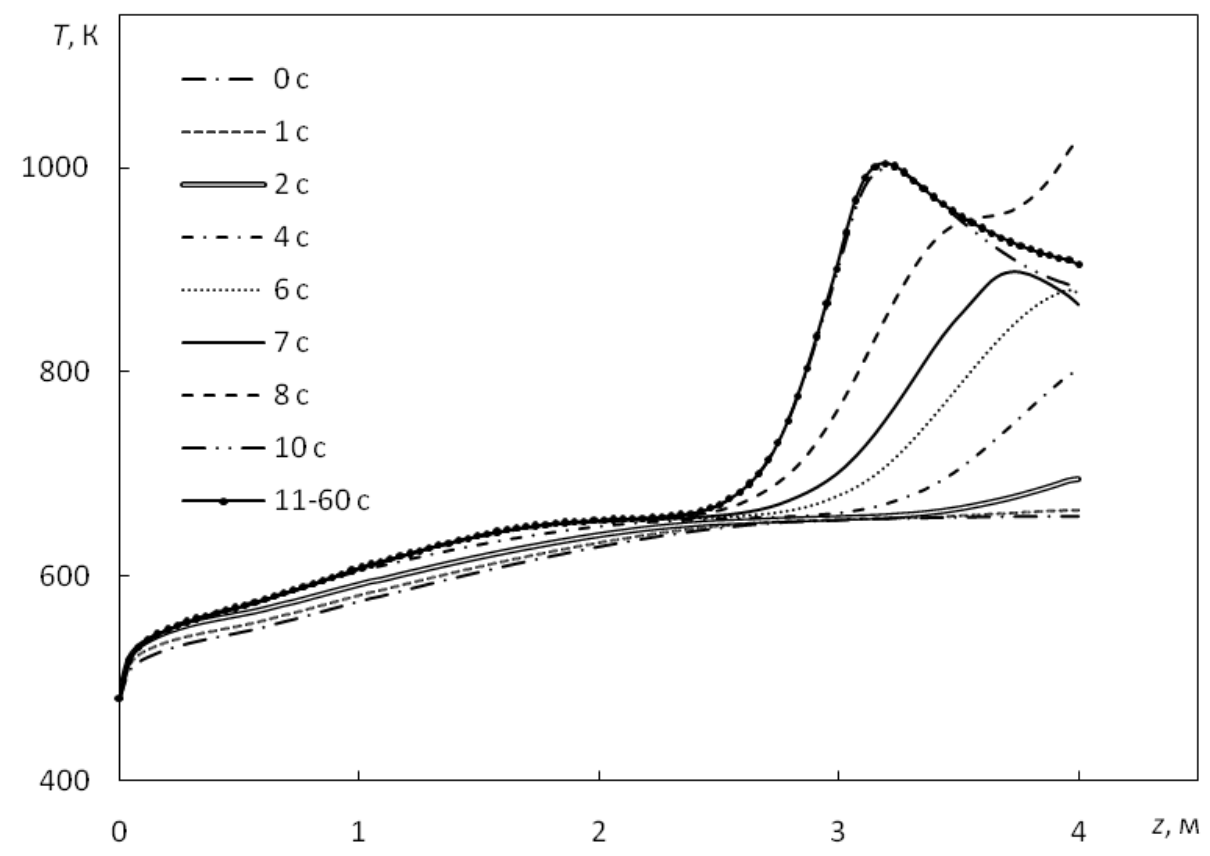

Рис. 1. Изменение во времени температуры стенки по длине трубы.

В исходном состоянии реализуется режим нормального теплообмена с равномерным увеличением температуры стенки по длине трубы. Начиная с 4 с, наблюдается формирование режима ухудшенного теплообмена с резким увеличением температуры стенки вблизи выходного сечения. Далее температура стенки увели- чивается, максимум температуры сдвигается к входному сечению. В конце переходного процесса, длительность которого составляет $11 \mathrm{c}$, максимальное значение температуры стенки находится в сечении 3 м от входа. На рис. 2, 3 представлено изменение во времени профиля скорости теплоносителя в сечениях 3 и 4 м от входа. 


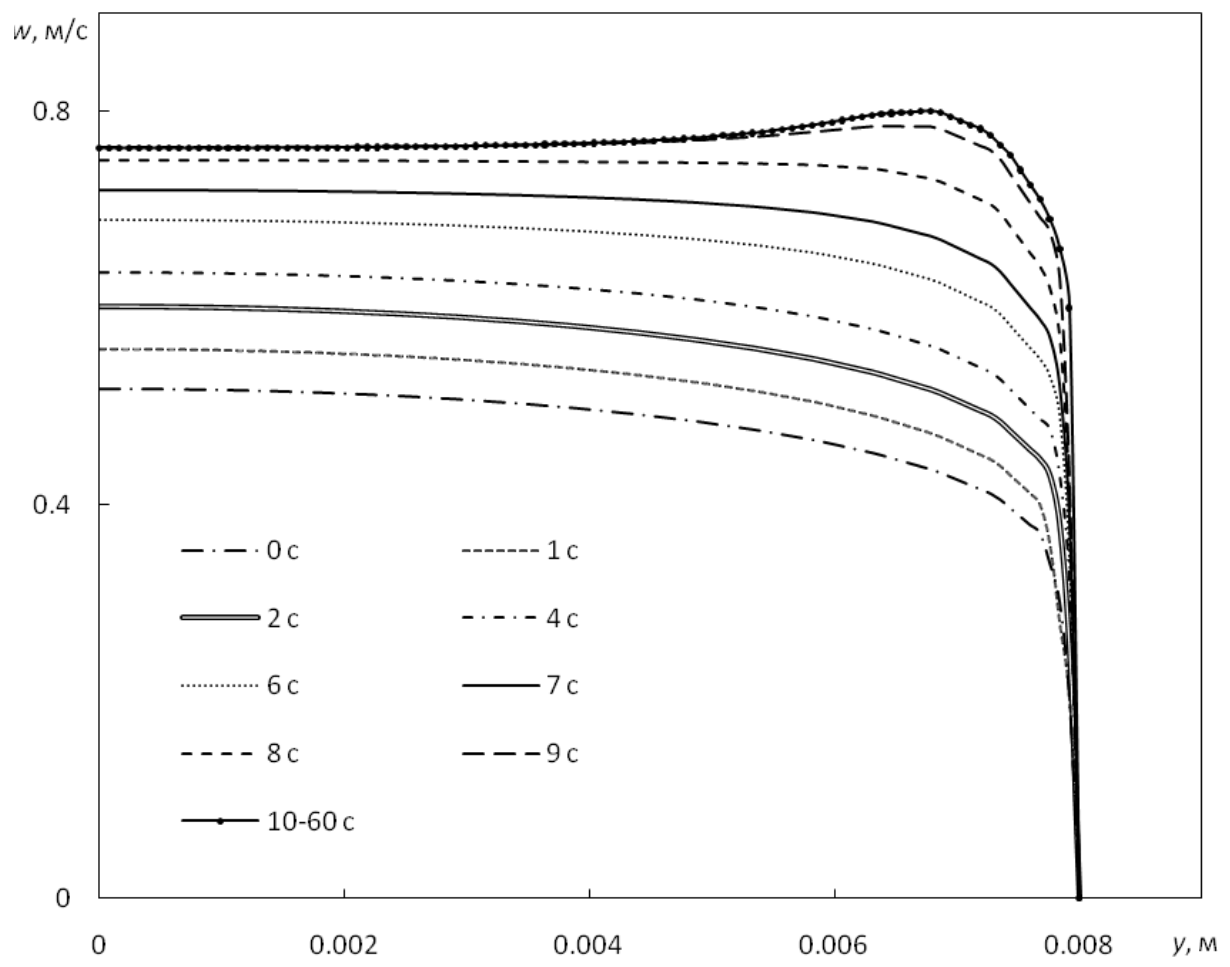

Рис. 2. Изменение профиля скорости в сечении 3 м от входа.

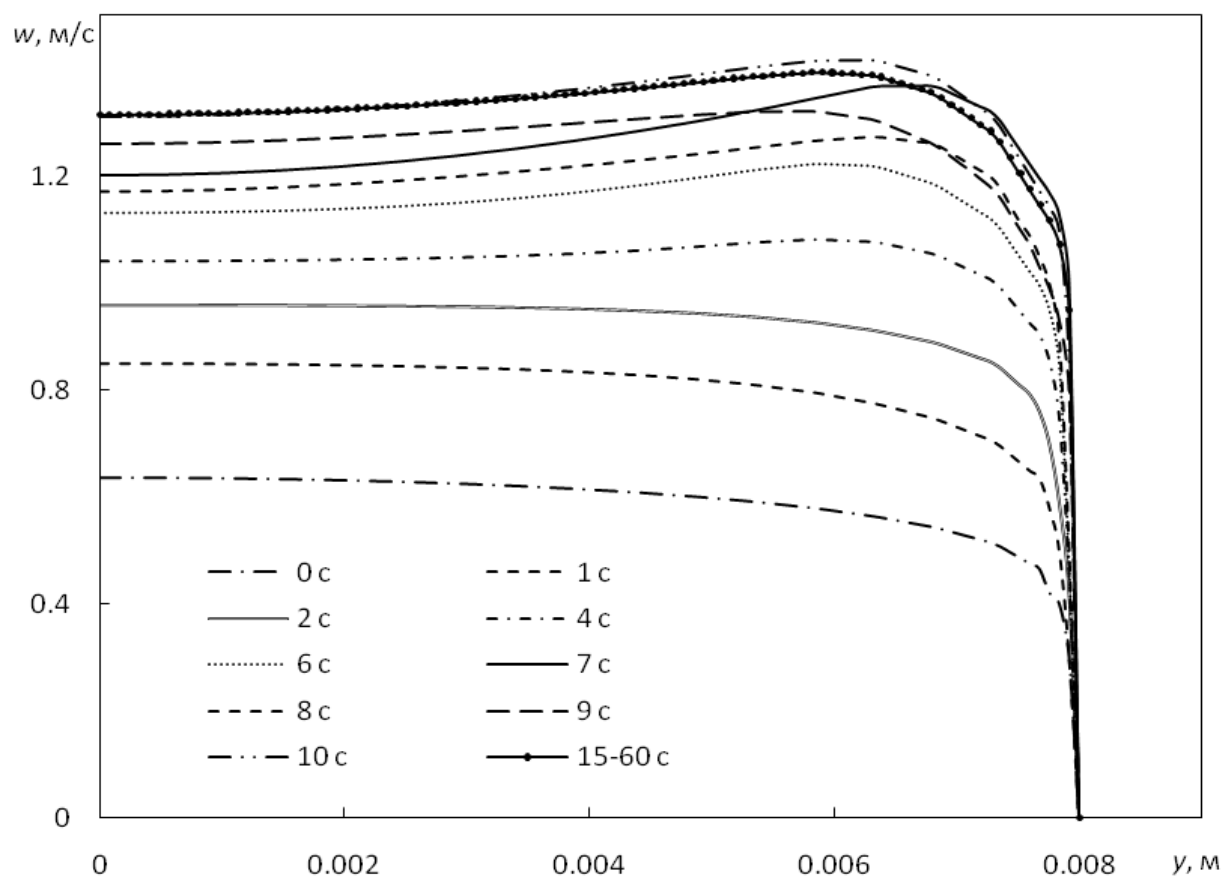

Рис. 3. Изменение профиля скорости в сечении 4 м от входа. 
В сечении 3 м от входа профиль скорости равномерно увеличивается и на 9 с приобретает М-образную форму с максимумом вблизи стенки. Это соответствует формированию режима ухудшенного теплообмена в этом сечении с максимальным значением температуры стенки (рис. 1) и температуры теплоносителя. В выходном сечении (рис. 3) картина другая. Уже на 6 с формируется М-образный профиль скорости, что приводит к резкому увеличению температуры стенки (рис. 1). На 7 с наблюдается резкое увеличение скорости как вблизи стенки, так и в середине трубы, а максимум температуры стенки перемещается в сечение 3,6 м. На 8 с наблюдается существенное уменьшение максимального значени скорости в выходном сечении, что отражается на профи- ле температуры теплоносителя (вблизи стенки температура резко увеличивается). При этом температура стенки имеет перегиб в сечении 3,6 м и резко увеличивается в выходном сечении (рис. 1). На 9 с наблюдается увеличение скорости в середине трубы в выходном сечении, профиль скорости становится более равномерным (максимум вблизи стенки «размазывается»), турбулентные пульсации из середины потока проникают к стенке и температура стенки падает. Зона ухудшенного теплообмена сдвигается к сечению 3 м от входа (рис. 1), где в это время формируется М-образный профиль скорости. Процесс стабилизируется после 11 с. На рис. 4 представлено изменение во времени коэффициента теплоотдачи по длине трубы.

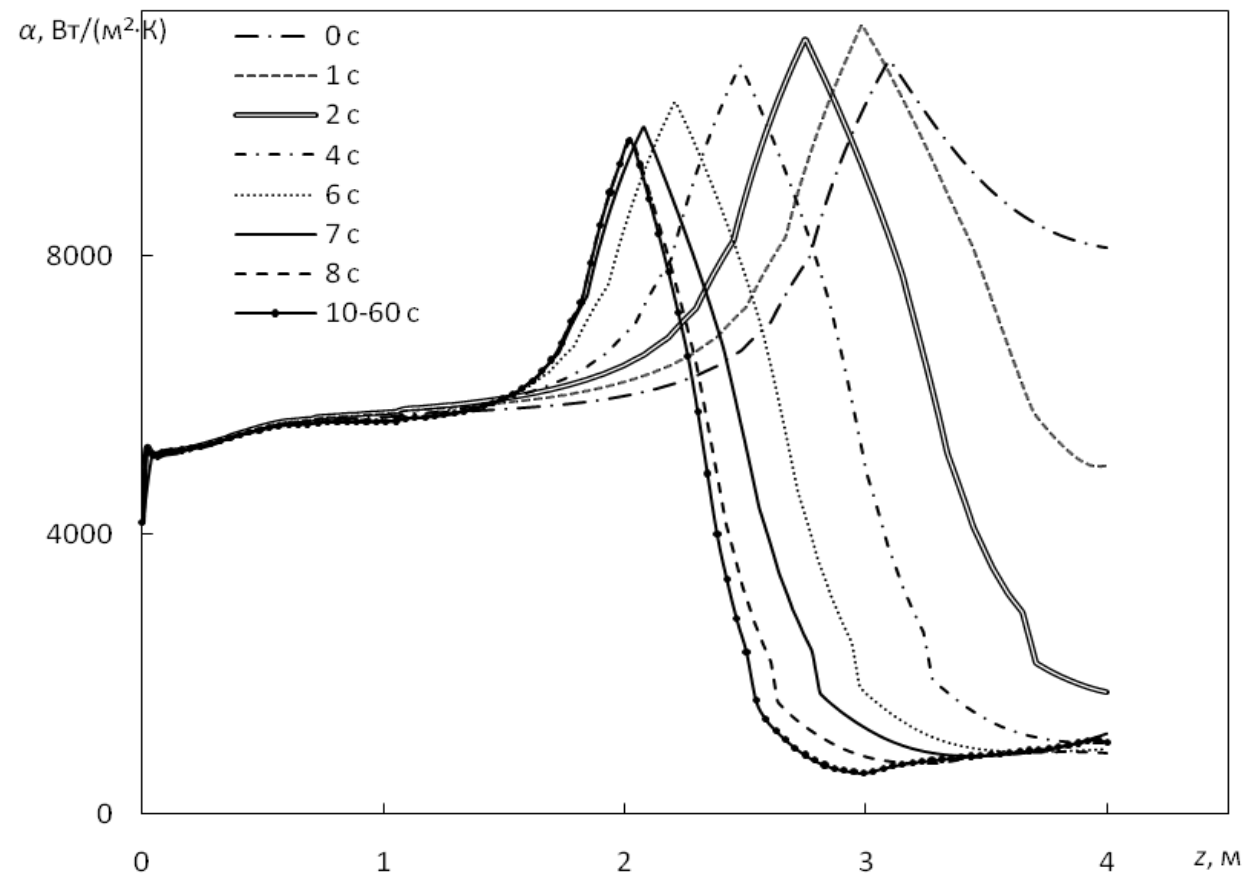

Рис. 4. Изменение во времени коэффициента теплоотдачи по длинне трубы.

В области резкого увеличения теплоемкости теплоносителя наблюдается увеличение коэффициента теплоотдачи, который достигает максимального значения при переходе к сверхкритической температуре. Резкое уменьшение коэффициента теплоотдачи вызвано изменением структуры потока, термическим ускорением в пристенной области, снижением теплопереноса от стенки в середину потока, формированию области ухудшенного теплообмена в соответствующем сечении. Смещение области ухудшенного теплообмена к входному сечению сопровождается смещением максимума и минимума коэффициента теплоотдачи в том же направлении. Интересно рассмотреть как протекает обратный процесс перехода от сверхкритических пара- 
метров к докритическим в результате снижения тепловой мощности.

\section{Уменьшение тепловой нагрузки}

В режиме с уменьшением тепловой нагрузки начальное значение плотности теплового потока на стенке трубы 400 кВт/м². В течении
2 с она падает до значения 300 кВТ/. $\mathrm{M}^{2}$ и далее не меняется. Параметры теплоносителя на входе в трубу такие же, как в режиме с увеличением тепловой нагрузки. На рис 5 показано изменение во времени температуры стенки по длине трубы.

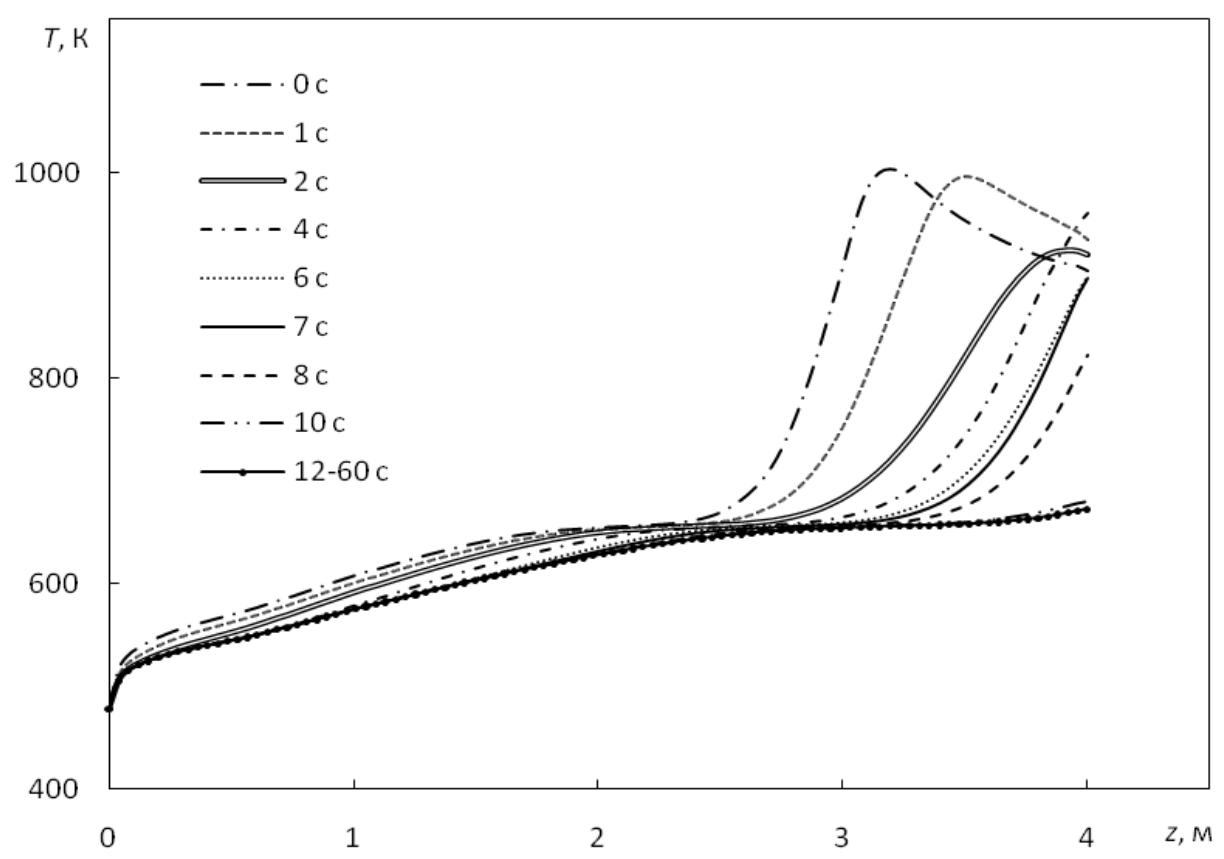

Рис. 5. Изменение во времени температуры стенки по длине трубы.

За 11 с наблюдается переход от режима ухудшенного теплообмена к нормальному режиму. Переход происходит более плавно по сравнению с предыдущим режимом. Максимум температуры сдвигается вниз по потоку от сечения 3 м от входа к выходному сечению и после 8 с резко уменьшается. Область ухудшенного теплообмена от сечения 3 м перемещается к выходному сечению и после 10 с вовсе исчезает.

На рис.6, 7 представлено изменение во времени профилей скорости в сечениях 3 и 4 м от входа. В связи с уменьшением плотности теплового потока на стенке скорость в трубе уменьшается. В сечении 3 м (рис. 6) профиль скорости изменяется от М-образного в начальный момент времени до равномерного в конце процесса. В сечении 4 м через 4 с профиль скорости деформируется становится М-образным с максимумом вблизи стенки, что соответствует максимуму температуры стенки в этом сечении (рис. 5). Далее скорость вблизи стенки уменьшается быстрее, чем в середине канала, максимум скорости смещается к центру канала.

Изменение во времени коэффициента теплоотдачи по длине трубы представлено на рис. 8.

В режиме с падением нагрузки минимум и максимум коэффициента теплоотдачи сдвигаются к выходному сечению. Если сравнить рис. 8 с рис. 4 , то видно, что процесс изменения коэффициента теплоотдачи проходит в обратном направлении. Однако конец процесса в режиме падения нагрузки не совпадает с началом процесса в режиме наброса мощности. Минимум коэффициента теплоотдачи в конце процесса на рис. 8 существенно меньше, чем на рис. 4 в начале процесса, что свидетельствует о небольшом гистерезисе процесса перехода к сверхкритической температуре и обратно. 


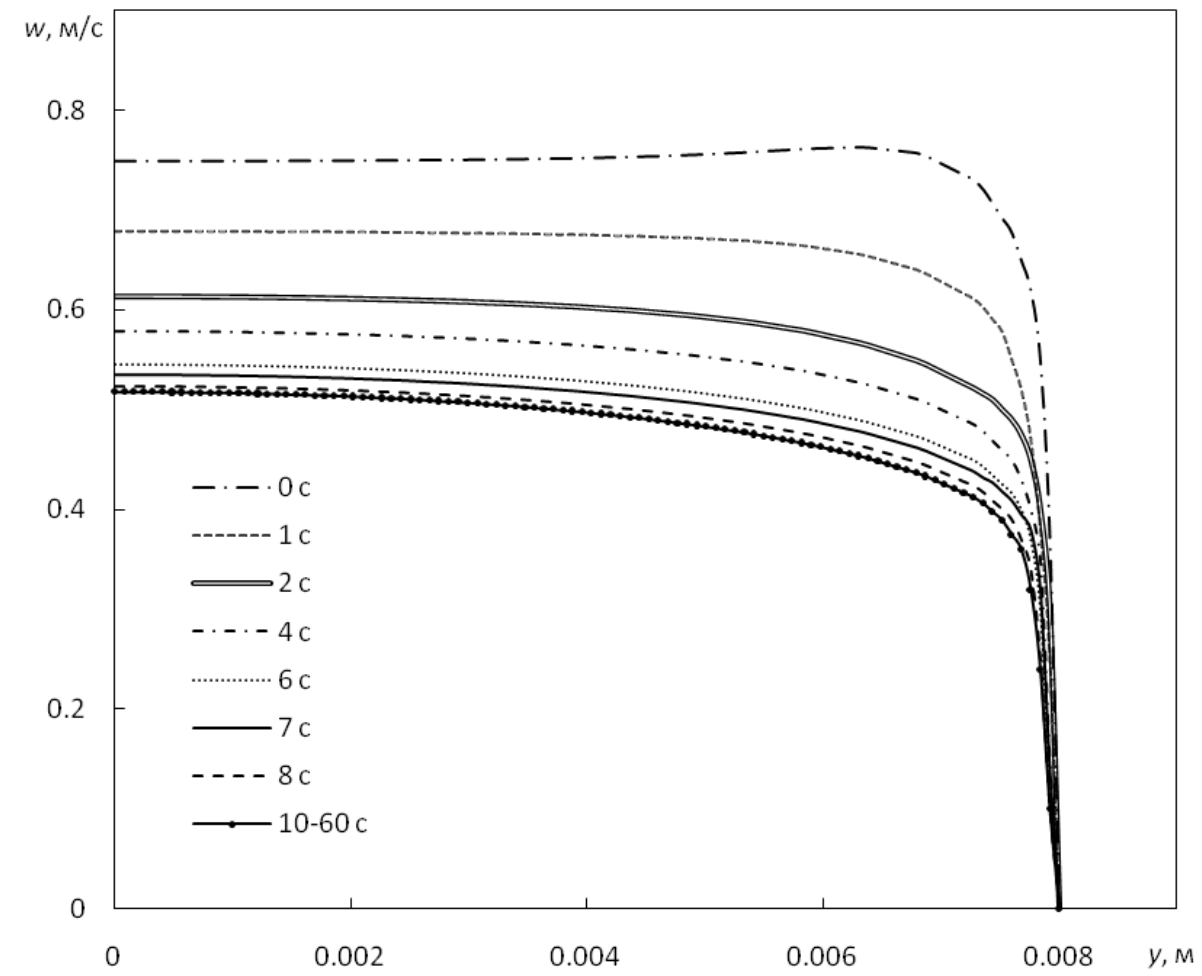

Рис. 6. Изменение профиля скорости в сечении 3 м от входа.

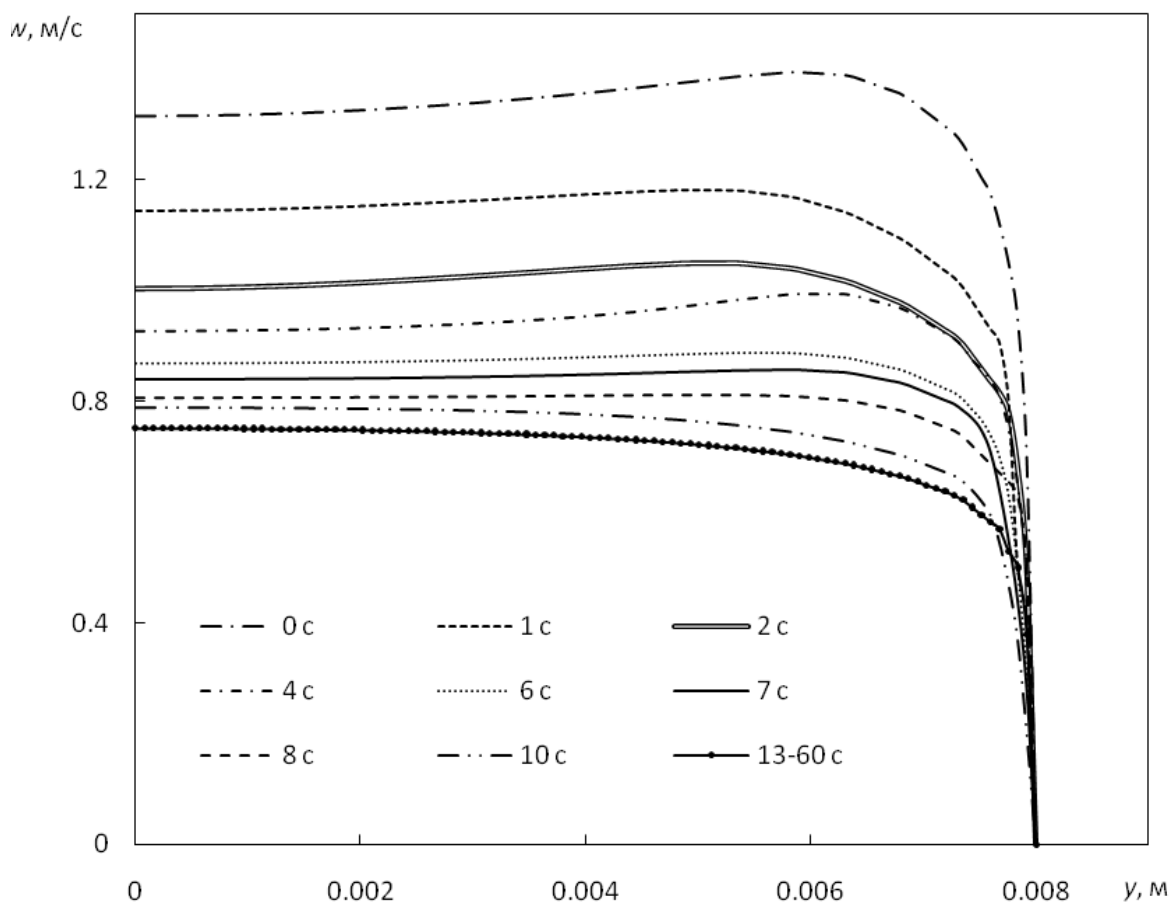

Рис. 7. Изменение профиля скорости в сечении 4 м от входа. 


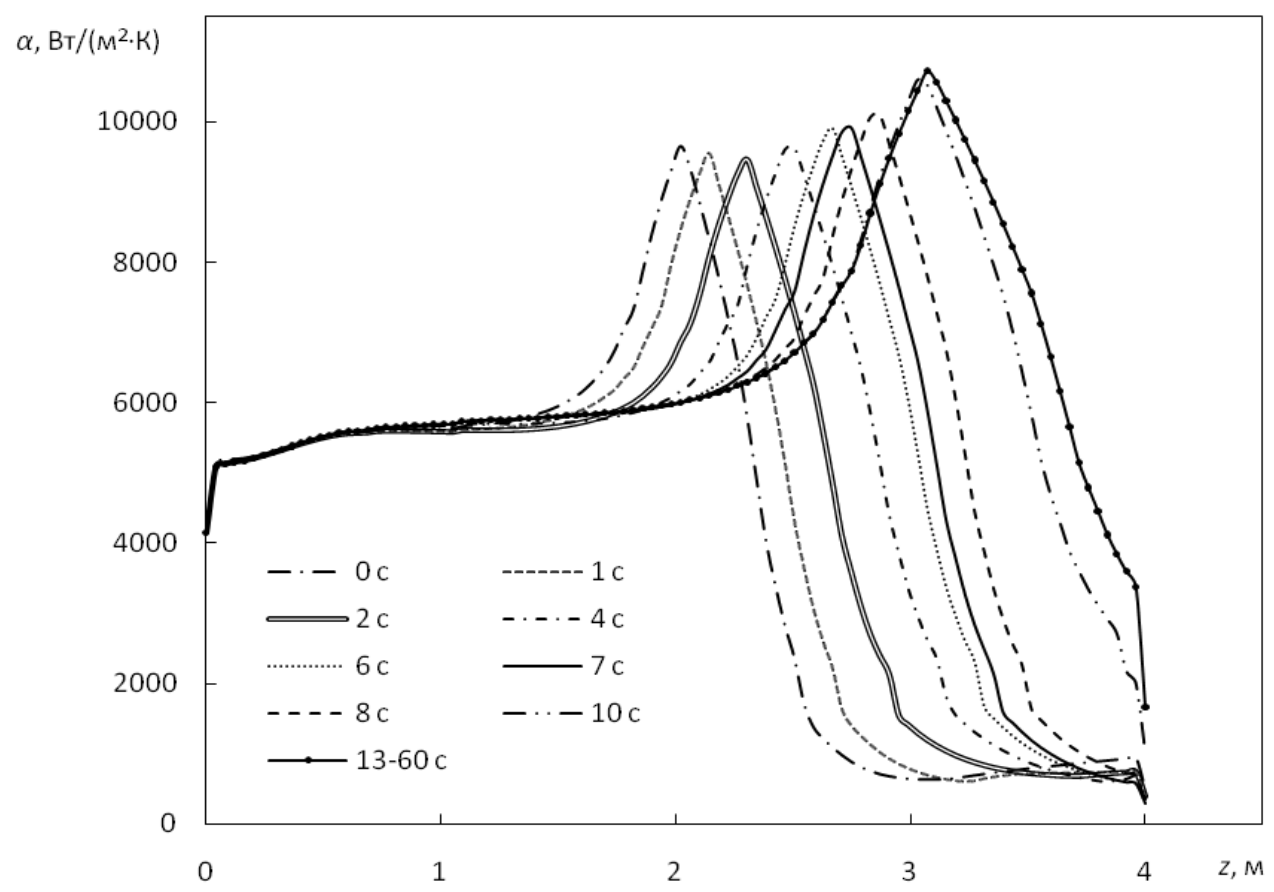

Рис. 8. Изменение во времени коэффициента теплоотдачи по длинне трубы.

\section{Выводы}

1. С увеличением плотности теплового потока профиль скорости существенно деформируется, приобретает М-образную форму с максимумом вблизи стенки. Возникновение режимов ухудшенного теплообмена связано с изменением структуры потока, существенной деформацией профиля скорости, вызванной термическим ускорением потока вблизи стенки, что существенно снижает отвод теплоты от стенки в ядро потока.

2. Длительность переходных процессов как при увеличении, так и при уменьшении мощности практически совпадает и составляет $11 \mathrm{c}$. Переход от режима ухудшенного теплообмена к нормальному режиму происходит более плавно, по сравнению с переходом от нормального режима к режиму ухудшенного теплообмена. При обратном переходе к режиму нормального теплообмена коэффициент теплоотдачи полностью не восстанавливается (минимум значительно ниже), что свидетельствует о незначительном гистерезисе переходного процесса.

\section{ЛИТЕРАТУРА}

1. Кириллов П.Л. Водоохлаждаемые реак- торы на воде сверхкритических параметров// Теплоэнергетика. - 2008. - №5. - С.2-5.

2. Cao L., Oka Y., Ishiwatari Y., Shang Z. Core Design and Subchannel Analysis of a Superfast Reactor// Journal of Nuclear Science and Technology. - 2008. - v.45, № 2. - P.138-148

3. Власенко Н.И., Годун О.В., Кирьянчук В.Н. Сравнительная оценка инновационных вариантов открытого ядерно-топливного цикла в Украине//Ядерна та радіаційна безпека. - 2014. T.3,№63. -C.10-13.

4. Pioro I.L., Khartabil H.F., Duffey R.B. Heat transfer to supercritical fluids flowing in channels empirical correlation (survey)// Nucl. Eng. Design. - 2004. - v.230. - P.69-91

5. Грабежная В.А., Кириллов П.Л. Теплообмен при сверхкритических давлениях и границы ухудшения теплообмена// Теплоэнергетика.-2006. - №4. - С.46-51.

6. Yang X., Su G.H., Tian W, Wang J., Qiu $S$. Numerical study on flow and heat transfer characteristics in the rod bundle channels under super critical pressure condition// Annals of Nuclear Energy. - 2010. - 37. - P.1723-1734

7. Gu Y.Y., Cheng X., Yang Y.H. CFD analysis of thermal - hydraulic behavion of supercritical water 
in sub -channels// Nucl. Eng. Des.- 2010. - 240(2). - P.364-374

8. Силин В.А., Семечков Ю.М., Алексеев П.Н., Митькин В.В. Исследование теплообмена и гидравлического сопротивления при течении воды сверхкритических параметров применительно к реакторным установкам// Атомная энергия. -2010 . - т.108, №6. - С.340-347

9. Курганов В.A. Теплообмен в трубах при сверхкритических давлениях теплоносителя// Труды РНКТ-4. - 2006. - т.1. - С.74-83

10. Авраменко А.А., Ковеикая М.М., Кондратьева Е.А., Тыринов А.И. Исследование режима ухудшенного теплообмена при течении воды сверхкритических параметров в трубе// Промышленная теплотехника. - 2015. -т.37, №1. C. 29

11. Алексеев Г.В., Силин В.А., Смирнов А.М., Субботин В.И. Исследование температурных режимов стенки трубы при теплосъеме водой сверхкритического давления// Теплофизика высоких температур.- 1976. - т.14, №4. - С.769774

12. Александров А.А., Орлов К.А., Очков В.Ф. Теплофизические свойства рабочих веществ теплоэнергетики. М.: Изд. Дом МЭИ. - 2009. $224 \mathrm{c}$. 


\section{HEAT TRANSFER OF SUPERCRITICAL PRESSURE FLOW IN PIPE WITH CHANGING THERMAL LOAD}

\section{A.A. Avramenko, M.M. Kovetskaya, E.A. Kondratieva, A.I. Tyrinov}

Institute of Engineering Thermophysics of the National Academy of Sciences of Ukraine, 03057, Kiev, vul. Zhelyabova 2a, Ukraine.

This paper analyzes the processes of heat transfer for the flow of supercritical water in the vertical heated pipe. Numerical study of the processes was based on the $k-\varepsilon$ RNG turbulence model. The results of simulations allow analyzing thermophysical and hydrodynamic character. The results of numeral simulations of heat transfer processes in nonstationary conditions with a modification of thermal loading are presented.

Key words: numerical modeling, vertical pipe, supercritical pressure.

References 12, fig. 8.

1. Kirillow P.L. Water cooled reactors on water of supercritical parameters // Teploenergetika. 2008. - №5. - P.2-5.(Rus.)

2. Cao L., Oka Y., Ishiwatari Y., Shang Z. Core Design and Subchannel Analysis of a Superfast Reactor// Journal of Nuclear Science and Technology. - 2008. - V.45, № 2. - P.138-148.

3. Vlasenko N.I., Godun O.V., Kiryanchuk V.N. Comparative estimation of innovative variants of an open nuclear-fuel cycle in Ukraine // Nuclear and radiating safety. - 2014. - V.3, №63 . - P.10-13. (Rus.)

4. Pioro I.L., Khartabil H.F., Duffey R.B. Heat transfer to supercritical fluids flowing in channels - empirical correlation (survey)// Nucl. Eng. Design. - 2004. - V.230. - P. 69-91.

5. Grabezhnaya V.A., Kirillow P.L. Heat exchange at supercritical pressure and borders of deterioration of heat exchange // Teploenergetika. 2006. - №4. - P.46 - 51.(Rus.)

6. Yang X., Su G.H., Tian W, Wang J., Qiu $S$. Numerical study on flow and heat transfer characteristics in the rod bundle channels under super critical pressure condition // Annals of Nuclear Energy. - 2010. - №37. - P. 1723-1734.

7. Gu Y.Y., Cheng X., Yang Y.H. CFD analysis of thermal - hydraulic behavior of supercritical water in sub -channels // Nucl. Eng. Des. . - 2010 . - V.240, №2. - P. 364-374.

8. Silin V.A., Semechkov Yu. M., Alekseev P.N., Mitkin V.V. Research of heat exchange and hydraulic resistance at a water current supercritical parameters with reference to реакторным to installations // Nuclear energy. - 2010. - V.108, №6. - P. 340 - 347. (Rus.)

9. Kurganov V.A. Heat exchange in pipes at supercritical pressure of the heat-carrier // The works RNKT - 4. - 2006. - V. - P.74-83. (Rus.)

10. Avramenko A.A., Kovetskaya M.M., Kondratieva E.A., Tyrinov A.I. Research of modes of the worsened heat transfer for the flow of supercritical parameters in the vertical pipe // Promyshlennaya teploteknika. - 2015. - V.37, №1. - P.29 34.(Rus.)

11. Alekseev G.V., Silin V.A., Smirnov A.M., Subbotin V.I. Research of temperature modes of a wall of a pipe at теплосьёме water of supercritical pressure // Thermophysics of high temperatures. 1976. - V.14, №4. - P. 769-774.(Rus.)

12. Aleksandrov A.A., Orlov K.A., Ochkov V.F. Thermal properties of working substances of power system. M.: MEI. - 2009. - 224p.

Получено 27.10.2015 Received 27.10.2015 